Laser Wakefield Acceleration at Reduced Density in the Self-Guided Regime

J. E. Ralph, F. Albert, S. H. Glenzer, J. P. Palastro, B. B. Pollock, J. L. Shaw, A. Till, D. H. Froula, C. E. Clayton, W. Lu, W. B. Mori, A. E. Pak, C. Joshi, S. Martin, L. O. Silva

November 20, 2009

Physics of Plasmas 
This document was prepared as an account of work sponsored by an agency of the United States government. Neither the United States government nor Lawrence Livermore National Security, LLC, nor any of their employees makes any warranty, expressed or implied, or assumes any legal liability or responsibility for the accuracy, completeness, or usefulness of any information, apparatus, product, or process disclosed, or represents that its use would not infringe privately owned rights. Reference herein to any specific commercial product, process, or service by trade name, trademark, manufacturer, or otherwise does not necessarily constitute or imply its endorsement, recommendation, or favoring by the United States government or Lawrence Livermore National Security, LLC. The views and opinions of authors expressed herein do not necessarily state or reflect those of the United States government or Lawrence Livermore National Security, LLC, and shall not be used for advertising or product endorsement purposes. 
APS/123-QED

\title{
Laser Wakefield Acceleration at Reduced Density in the Self-Guided Regime
}

\author{
J.E. Ralph, F. Albert, S. H. Glenzer, J. P. Palastro, \\ B. B. Pollock, J. L. Shaw, A. Till, and D. H. Froula \\ Lawrence Livermore National Laboratory, Livermore, CA 94550, USA \\ C. E. Clayton, W. Lu, W. B. Mori, A. E. Pak, and C. Joshi \\ Department of Electrical Engineeing, \\ UCLA, Los Angeles, CA 90095, USA \\ S. Martin and L. O. Silva \\ GoLP/Instituto Plasmas e Fusa o Nuclear, \\ Instituto Superior Te cnico, Lisbon, Portugal
}

(Dated: November 18, 2009)

\begin{abstract}
Experiments conducted using a 200TW 60 fs laser have demonstrated up to $720 \mathrm{MeV}$ electrons in the self-guided laser wakefield regime using pure Helium gas jet targets. Charge and energy of the accelerated electrons was measured using an electron spectrometer with a $0.5 \mathrm{~T}$ magnet and charge callibrated image plates. The self-trapped charge in a helium plasma was shown to fall off with decreasing electron density with a threshold at $2.5 \times 10^{18}\left(\mathrm{~cm}^{-3}\right)$ below which no charge is trapped. Self-guiding however is shown to continue below this density limitation over distances of $14 \mathrm{~mm}$ with an exit spot size of $25 \mu \mathrm{m}$. Simulations show that injection of electrons at these densities can be assisted through ionization induced trapping in a mix of Helium with $3 \%$ Oxygen.
\end{abstract}

PACS numbers: 52.38.Kd, 41.75.Jv, 52.35.Mw 


\section{INTRODUCTION}

It has been more than 30 years since the first paper introducing the laser plasma accelerator was published[1]. The promise of producing a tabletop electron accelerator is on the verge of becoming reality. Such laser wakefield accelerators (LWFAs) have been shown to accelerate electrons with gradients exceeding $100 \mathrm{GeV} / \mathrm{m}[2]$. Gradients in the range of $50 \mathrm{MeV} / \mathrm{m}$ are typical for current radio frequency accelerators requiring long distances to obtain high energies.

Experimental results from laser wakefield accelerators have demonstrated that high energy and high quality electron beams can be produced. In 2004, three groups independently produced electrons beam from gas jet targets with electron plasma densities $\left(n_{e}\right)$ near $10^{19}$ $\mathrm{cm}^{-3}[3-5]$. Electron energies produced in these experiments were in the range of $200 \mathrm{MeV}$. A $1 \mathrm{GeV}$ beam was later reported[6] using a discharge driven capilary plasma channel to guide the laser pulse.

Producing high quality high energy electron beams without the need for an external guiding structure would greatly simplify a practical laser wakefield accelerator. Research in the self-guided regime has lead several important recent results. The physics of maintaining the high intensity of short laser pulses over distances significant for acceleration was demonstrated through self-guiding and shown to be limited by nonlinear pump depletion[7]. Others in this regime have demonstrated self-trapping of electrons with significant acceleration[8]. Very recently, high energy gain in the self-guided regime has produced energetic electron beam with energies exceeding $700 \mathrm{MeV}[9,10]$. Results outlined in this paper show that self-guiding does not limit electron energies to less than a $\mathrm{GeV}$, but that a limit on the self-trapping of charge at lower densities leads to no electrons accelerated to that energy.

\section{PHYSICAL PICTURE}

The blowout or bubble LWFA regime occurs when an ultrashort $c \tau<\lambda_{p} \approx 2 R_{B}$ and relativistically intense laser pulse $I>10^{18} \mathrm{~W}-\mathrm{cm}^{-2}$ propagates through an underdense plasma $\left(n_{e}<n_{c}\right)$. Here $\tau$ is the full-width-half-max duration of the laser pulse, $\lambda_{p}$ is the

classical plasma period, $R_{B}$ is the electron blowout radius, and $n_{c}$ is the critical density of $1.7 \times 10^{21} \mathrm{~cm}^{-3}$. The ponderomotive force of such a laser pulse expells electrons, leaving a 
region behind the pulse with an electron density nearly zero. The ions are motionless on this time scale but provide a uniform positive background charge which pulls the expelled electrons back toward the axis by coulomb attraction. The high longitudinal accelerating electric field is a result of this transient charge displacement moving with a velocity $v_{f}$ equal the group velocity of the driving laser pulse.

To maintain the relativistic intensity necessary for operating in the blowout regime over distances greater than a Rayliegh Length $Z_{R}=\pi w_{0}^{2} / \lambda_{0}$, the laser pulse must be guided in the plasma. The term $\lambda_{0}$ denotes the vacuum laser wavelength. Guiding of laser pulses occurs when diffraction is balanced by focusing. A plasma channel with a radial increase in density has the potential to guide a laser pulse if $\delta n / n_{e}\left(w_{0}\right)>\left[2 \omega_{p} /\left(c w_{0}\right)\right]^{2}[11]$. The term $\delta_{n}$ is the difference in on-axis electron density compared to the electron density at the $1 / e^{2}$ spot radius $w_{0} ; n_{e}$ is the electron density at radius, $w_{0}$. The vacuum speed of light was denoted c, and $\omega_{p}=\sqrt{n_{e} e^{2} /\left(\epsilon_{0} m_{e}\right)}$ is the electron plasma frequency. Here $\epsilon_{0}$ is the permitivity of free space, e is the charge of one electron and $m_{e}$ is the electron mass. In the blowout regime, most of the laser pulse resides in the bubble region in which the electrons have been expelled. An approximation of the guiding condition can be found for the portion of the pulse residing in the bubble by allowing $\delta n / n_{e}\left(w_{0}\right)=1$, resulting the reduced inequality $w_{0}>4 c / \omega_{p}$. Efficient self-guiding occurs because the front of the laser pulse locally gives it's energy to the wake before diffracting[12-14]. As the laser pulse tranfers energy to the blownout electrons, it etches back at a velocity $v_{\text {etch }}=c \omega_{p}^{2} / \omega 0^{2}$. This leads to a wake phase velocity $v_{f}=c-v_{\text {etch }}$. Electrons interacting with the front of the laser pulse are blown out rapidly by the ponderomotive force of the laser $F_{p} \propto a_{0}^{2}$. Here $a_{0} \approx 0.86 \sqrt{I_{0} \lambda_{0}^{2}}$ is the normalized vector potential of the laser pulse. Solving the nonlinear kinetic equation of motion of such electrons in the spead-of-light frame, $\frac{\partial p}{\partial t}=\frac{q}{m}(\mathbf{E}+\mathbf{v} \times \mathbf{B})+\mathbf{F}_{\mathbf{p}}$, results in a nearly circular trajectory with a radius $R_{B}=\left(2 \omega_{p} / c\right) \sqrt{a_{0}}[13]$. A matched self-guided LWFA starts with an initial $w_{0} \sim R_{B}$. This condition represents the steady state in which the radial variation in spot size is minimum. Larger spots with sufficient power will evolve to the matched state by self-focusing, while smaller spots are harder to predict[14-16].

Ionization occurs well ahead of the main laser pulse by tunnel ionization[17]. The temperature of electrons produced this way is negligible compared to the laser and wake potential. These cold electrons born at rest nonetheless have a large kinetic energy, K.E., in the frame moving with velocity $v_{f}$. An electron born in a neutral plasma is born with initial poten- 


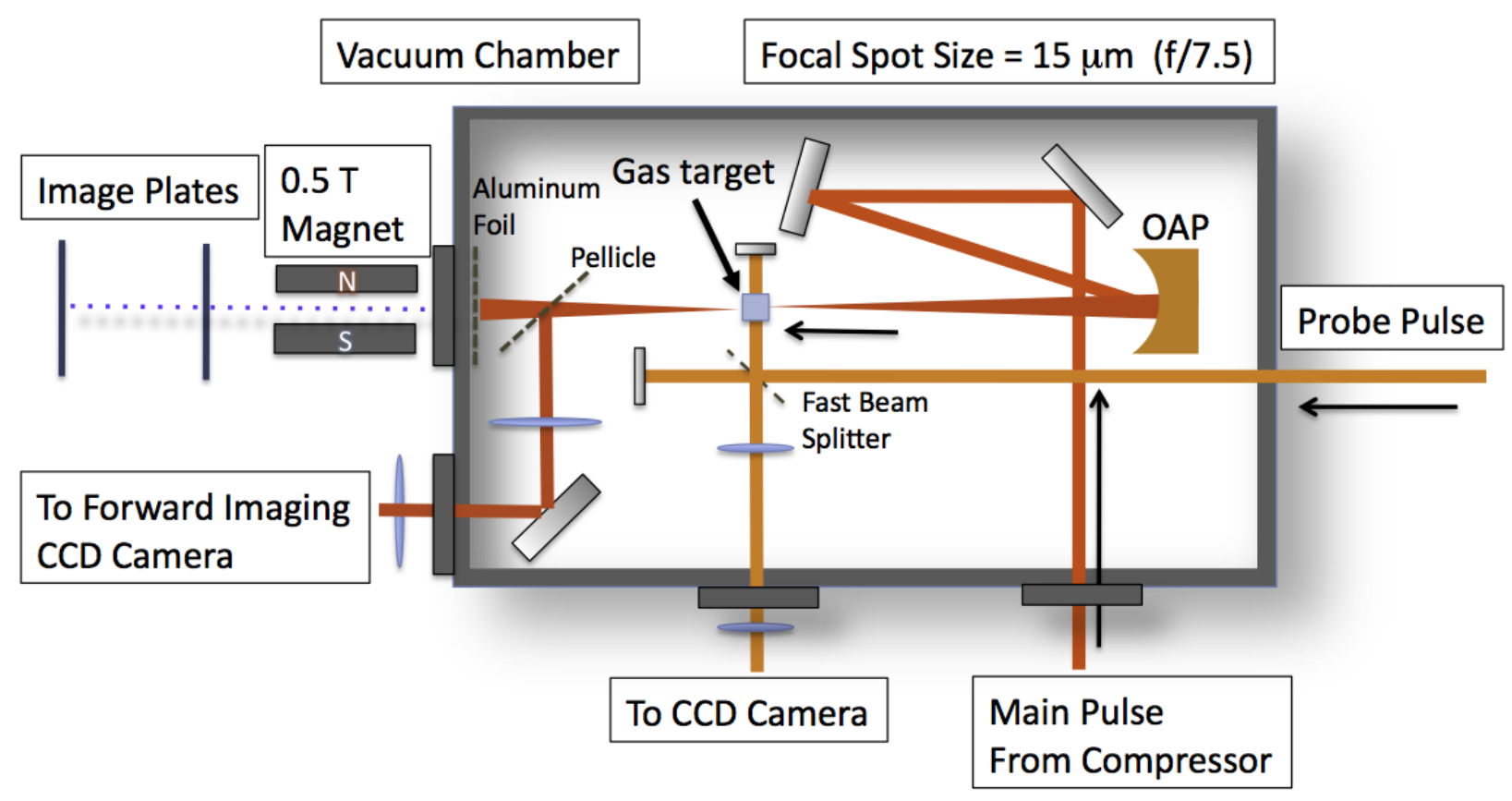

FIG. 1: A schematic of the experimental setup. The three primary diagnostics are shown: forward electron spectrometer utilizing the $0.5 \mathrm{~T}$ permanent magnet, Michelson interferometry, and a forward image of the spot at the exit plane.

tial $\phi=0$. The electron interacts with the laser goes down the potential hill where it is attracted to the ions and continues up the potential hill at the back of the bubble where the blownout electrons are converging on-axis. When the potential at the back of the bubble is higher than the initial kinetic energy K.E., the electron will be trapped. These self-injected electrons are accelerated from the back of the bubble and have a velocity of approximately c. The trapped electrons move with a velocity c higher than the accelerating structure (or bubble) which moves at $v_{f}$. Since the accelerating force of the bubble extends to the center, trapped electrons will gain energy until they reach the center. In the lab frame, electrons can gain energy over a length known as the dephasing length given by,

$$
L_{d p}=\frac{c}{\left(c-v_{f}\right)} R_{B} \approx \frac{2 \omega_{0}^{2}}{3 \omega_{0}^{2}} \propto \frac{1}{n_{e}}
$$

If the length of the plasma is matched to the dephasing length, a maximum energy can be found by,

$$
E_{\max }=\left(\frac{P}{T W}\right)^{1 / 3}\left(\frac{10^{18} \mathrm{~cm}^{-3}}{n_{e}}\right)^{4 / 3}
$$


This equation provides a means to compare the maximum energy of accelerated electrons as a function of density and power only, provided the length is set appropriately.

Through the physics outlined in this section, a comparison of the effect of density can be drawn which will aid in the understanding of the experimental results. In summary, raising the density helps self-guiding since the effective density channel is larger. Also a higher density will allow easier self-trapping as explained by our simple physical picture which shows a slower phase velocity which results in a smaller initial electron kinetic energy and therefore easier trapping. Lowering the density however is necessary to achieve high energies because dephasing takes longer to occur at lower densities. Experiments described in the following section show progress in addressing self-guiding and self-injection as the density is lowered to achieve higher energy.

\section{EXPERIMENTAL MEASUREMENTS}

Experiments were conducted at the Jupiter Laser Facility at Lawrence Livermore National Laboratory, using the Callisto Laser System. The Callisto laser system is a Ti:Sapphire laser with a central wavelength $\mid a m b d a_{0}$ of $806 \mathrm{~nm}$. It delivers up to $18 \mathrm{~J}$ on target in a 60 fs laser pulse providing up to $300 \mathrm{TW}$ each laser pulse. Typical data shots were between 6 and 14J. The rep rate of the high power is 2 shots per hour. The spot size, $w_{0}$, of the laser pulse after focusing with an $\mathrm{f} / 8$ Off-Axis Parabolic Mirror (OAP) was $15 \mu \mathrm{m}$ radius $1 / e^{2}$ intensity. The central spot contained approximately 30\% of the total laser energy[10]. Comparisons with theory and simulations include only the energy in the central spot.

Following the experimental setup shown in Fig.(1), three primary diagnostics were utilized. An electron spectrometer using a $0.5 \mathrm{~T}$ permanent magnet and 2 forward image plates was used to diagnose the electron energy and determine the electron charge[10, 18]. The transmitted light was collected and an image of the exit of the of the gas target was relayed to a 12 bit CCD camera to diagnose the quality of the self-guiding. Additionally, a fast Michelson type interferometer was used to determine the plasma density prior to hydrodynamic expansion.

The interferometer used was a single pass Michelson. To achieve only a single pass using a Michelson Interferometer, a probe pulse was split from the main pulse and compressed to approximately 100 fs. The 100 fs pulse was allowed to pass through the gas target prior 


\section{gas jet range}

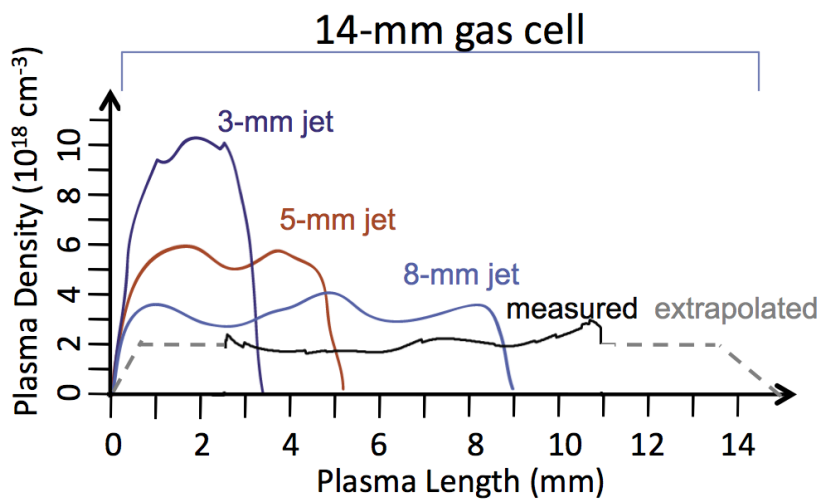

FIG. 2: Plasma Density profiles from the the $3 \mathrm{~mm}, 5 \mathrm{~mm}$, and $8 \mathrm{~mm}$ gas jets and from the 14 $\mathrm{mm}$ long gas cell. Direct measurments of the density of the gas cell were limited to the window region. The dashed line shows the extrapolated region.

to the main pulse creating the plasma. The delay between the probe and the main pulse were timed so that the on the return trip the main pulse had passed through the target and formed the plasma. A typical probe delay was 3 ps. The resulting interferogram was than analyzed and Abel-inverted to determine a density map.

Four gas target were used in the experiments. Supersonic gas jets of length 3, 5 and $8 \mathrm{~mm}$ provided uniform electron plasma densities ranging from 2 to $10 \times 10^{18}\left(\mathrm{~cm}^{-3}\right)$ as shown in Fig. 2[19]. A gas cell target has been developed for lengths longer than $\sim 1 \mathrm{~cm}$ with densities less than $3 \times 10^{18}\left(\mathrm{~cm}^{-3}\right)$. A schematic of the gas cell target is shown if Fig. 2. The cell used in the experiments had a total length of $14 \mathrm{~mm}$ from pinhole to pinhole. The entrance pinhole used in the experiments, has a diameter of $500 \mu \mathrm{m}$ while the exit has a diameter 2 $\mathrm{mm}$. A uniform density could be seen over the $9 \mathrm{~mm}$ probe window range. A ramp up and ramp down are assumed to be approximately the same size as the diameters of the pinholes used. This is shown also in Fig. 2 as the dashed curve. The density data provided here was taken from the density maps obtained from the transverse plasma interferometry.

The maximum energy of electrons as a function of density is shown in Fig. 3. This data has been taken using the gas jet targets described above and pure Helium gas. Here, the electron plasma density of the targets was varied to allow for the dephasing length to be maximized and therefore the electron energy. Under the conditiions used in these experiments, self-trapping required some evolution of the of the main pulse. The length was 


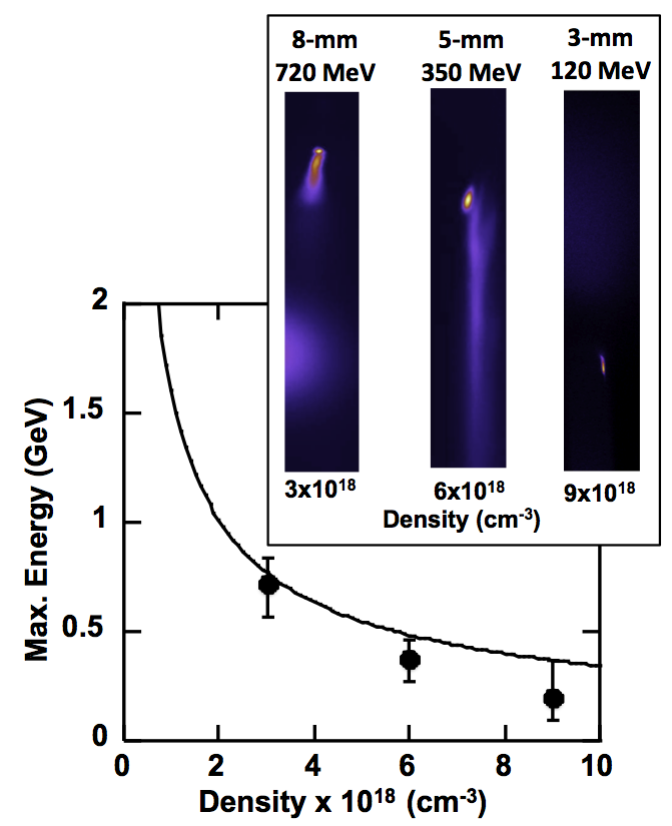

FIG. 3: Images of the raw images plates are shown along with a plot of the density vs. energy. The curve is a plot of equation 2 representing the maximum energy electrons at these densities.

determined to be $3 \mathrm{~mm}$ and was confirmed using 3D particle-in-cell simulations[10]. With this, the maximum energy curve Eqn. 2 could be superimposed as is shown in Fig. 3 allowing for the $3 \mathrm{~mm}$ prior to self-injection. From Eqn. 1 we can see that to achieve still longer dephasing lengths, a lower density is required. At the lowest density, a maximum energy was achieved of $720 \mathrm{MeV}$ in the $8 \mathrm{~mm}$ gas jet. Increasing the density, while decreasing the length in order to compensate for the shorter dephasing length of the plasma results in the expected trend.

Shown in Fig. 4 is the charge for shots with electron energies above $100 \mathrm{MeV}$ as a function of $n_{e}$. From the data, it is clear that self-trapping is more readily achieved at higher density. Furthermore, a threshold occurs at a density of $2.5\left(10^{18} \mathrm{~cm}^{-3}\right)$, below which no trapped charge is observed. It is expected that trapping is easier at high densities because the phase velocity of the bubble $v_{f}$ is lower and therefore the relative initial kinetic energy of the electrons is lower. However, insufficient self-guiding at such low densities may also result in reduced or non-existent self-trapping. Self-trapping relies on maintaining the strong longitudinal field created by the ponderomotive force of the high intensity guided pulse. As the density is decreased, the channel amplitude $\Delta n / n$ diminishes everywhere. It can be expected that at some point, the front of the laser pulse will continue to erode in the region 


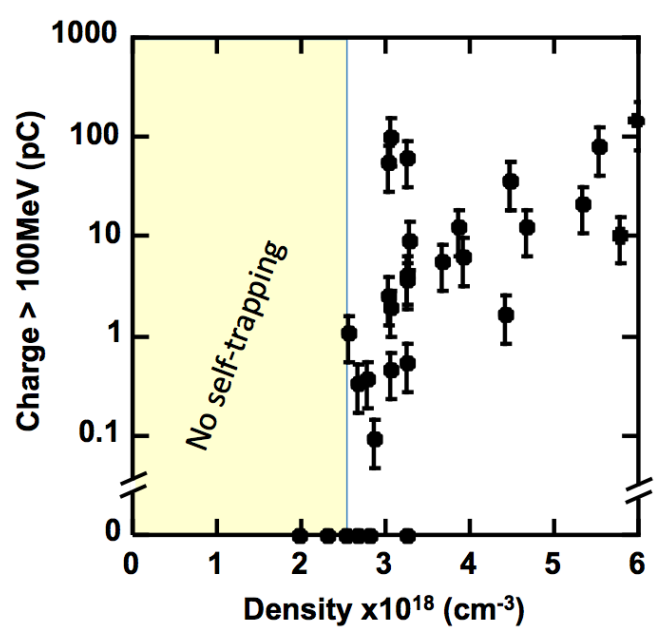

FIG. 4: Data showing a decrease in self-trapped charge with decreased density in a pure Helium plasma. A threshold is shown at approximately $2.5\left(10^{18} \mathrm{~cm}^{-3}\right)$ below which no trapped charge is observed.

where the blowout occurs resulting in a poorly guided pulse and therefore reduced intensity and a pure accelerating structure.

In Figs. 5a and 5b, two views of the exit of the $14 \mathrm{~mm}$ gas cell are shown. The initial $15 \mu \mathrm{m}$ laser focal spot was aligned so that the focus was centered in the same plane as the edge of the entrance pinhole. Rotation of the gas cell in both directions was used ensure that in vacuum, the laser propagated straight through and was centered on the exit $2 \mathrm{~mm}$ pinhole. Fig 5a. is an image of the vacuum spot at the exit plane of the gas cell and shows a diffracting spot with a spotsize of $250 \mu \mathrm{m}$. The 75 TW pulse appears well guided in the pure He plasma $n_{e}$ of $1.7 \times 10^{18}\left(\mathrm{~cm}^{-3}\right.$ over the distance of $14 \mathrm{~mm}$ with an exit spot size of $25 \mu \mathrm{m}$. The white curve in Fig. 5b shows a sum out over the entire chip along the vertical direction while the black curve shows a lineout through the peak. The spotsize of the exit spot was determined through a gaussian fit of the black curve with the reported spotsize being the $1 / e^{2}$ radius of this fit. This suggests that a strong wake exists throughout the plasma. The self-guided spot seen in Fig 5b is shown to be approximately $200 \mu \mathrm{m}$ below the vacuum spot indicating that the laser pulse does not follow the vacuum trajectory when helium is present. This is in agreement with the measured $-50 \mathrm{mR}$ deflection angle of the accelerated electrons in the gas jets seen on the forward image plates. We conclude that the gas jets themselves are not responsible for the deflection. At this density, no self-injection of electrons was seen on the forward image plates. Recently, results using ionization injection 

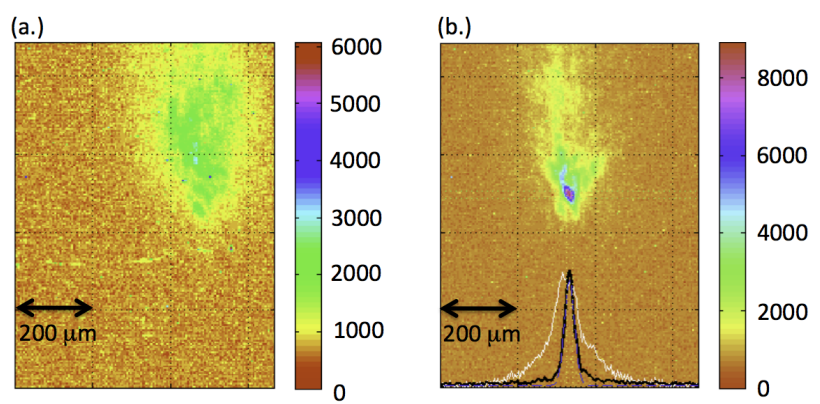

FIG. 5: An image of the exit of the $14 \mathrm{~mm}$ gas cell is shown for the case of vacuum (a) and with a pure Helium plasma with a density of $1.7 \times 10^{1} 8 \mathrm{~cm}^{-} 3$.

have demonstrated more consistent injection of electrons[20]. By adding small amounts of an impurity gas such as Nitrogen or Oxygen, the difference between the ionization levels of the k-shell and l-shell electrons can lead to an increase of injection of the k-shell electrons. The bi-gaussian shape of the laser pulse used in experiments results in ionization of the k-shell electrons at approximately $10^{19} \mathrm{~W}-\mathrm{cm}^{-} 2$. These electrons are created inside the bubble while the L-shell electrons are ionized early. The L-shell electrons contribute to the spherical bubble. This is very important to not lead to ionization induces defocusing of the laser pulse[]. The k-shell electrons however, are generated at rest inside the bubble at a lower potential. Electrons born at a lower potential have a larger potential barrier to overcome at the back of the wake in order to not become trapped. Oxygen was chosen as the mix-gas because the k-shell ionization level is very close to the peak intensity of the laser pulse at approximately $10^{19} \mathrm{~W}-\mathrm{cm}^{-2}$. This leads to electrons injected close to axis and therefore with reduced devergence and with a lower probability to radiate. Additionally, the large gap between the ionization energies of the k-shell and l-shell electrons in oxygen is sufficient to ensure that l-shell electrons are ionized early in the rise of the laser pulse and therefore contribute to the well-form bubble structure and do not lead to ionization induced defocusing which may make self-guiding more difficult.

Two dimensional Particle-in-cell simulations were conducted to look at ionization induced injection using parameters similar to the experimental conditions. The results of these simulations at a density of $2 \times 10^{18}\left(\mathrm{~cm}^{-} 3\right)$ are shown are shown in Fig. 6. In Fig 6a, the pure Helium shows a well form wake consistent with good self-guiding and maintaining a high intensity pulse but shows no trapped electrons. Fig 6b. shows the same simulation with mixture of $3 \%$ oxygen and $97 \%$ Helium. This simulation shows the same nice blowout 

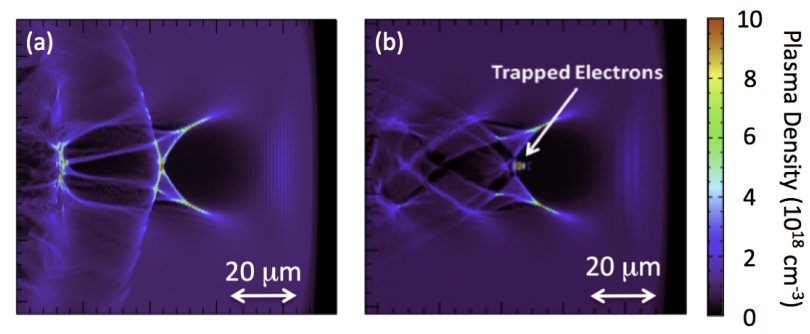

FIG. 6: Particle-in-cell simulations comparing the case of no oxygen vs. 3 percent oxygen

structure but with electrons trapped close to axis at the back.

Through this study, experiments have been performed which demonstrate a density threshold for the self-trapping of electrons in a pure helium plasma using a 75 TW laser pulse. This density threshold was found to be at $2.5 \times 10^{18}\left(\mathrm{~cm}^{-3}\right)$. This lower bound on self-injection was shown to limit the maximum energy of accelerated electrons to less than 1 $\mathrm{GeV}$. The maximum energy obtained through this study was $720 \mathrm{MeV}$ over a distance of 8 mm using a gas jet target. Studies continued over longer distances with lower densities using a gas cell target. In pure helium, at a plasma density of $2 \times 10^{18}\left(\mathrm{~cm}^{-3}\right)$, self-guiding was demonstrated over a distance of $14 \mathrm{~mm}$. However, no electrons were trapped. According to scalling, this could provide acceleration of trapped electrons beyond a GeV energy. To lower the threshold for trapping, a impurity of oxygen has been suggested. Particle-in-cell simulations have confirmed that this method can lower the density threshold and provide trapped electrons. This work performed under the auspices of the U.S. Department of Energy by Lawrence Livermore National Laboratory under Contract DE-AC52-07NA27344 and a Department of Energy Grant No. DEFG03-92ER40727 (UCLA) and was partially funded by the Laboratory Directed Research and Development Program under pro ject tracking code 06-ERD-056.

[1] T. Tajima and J. Dawson, Phys. Rev. Lett. 43, 267 (1979).

[2] C. Joshi, Physics of Plasmas 14, 055501 (pages 14) (2007).

[3] S. P. D. Mangles, C. D. Murphy, Z. Najmudin, A. G. R. Thomas, J. L. Collier, A. E. Dangor, E. J. Divall, P. S. Foster, J. G. Gallacher, C. J. Hooker, et al., Nature 431, 535 (2004).

[4] C. G. R. Geddes, C. Toth, J. van Tilborg, E. Esarey, C. B. Schroeder, D. Bruhwiler, C. Nieter, 
and J. C. W. P. Leemans, Nature 431, 538 (2004).

[5] J. Faure, Y. Glinec, A. Pukhov, S. Kiselev, S. Gordienko, E. Lefebvre, J. P. Rousseau, F. Burgy, and V. Malka, Nature 431, 541 (2004).

[6] W. P. Leemans, B. N. B, A. J. Gonsalves, C. Toth, K. Nakamura, C. G. R. Geddes, E. Esarey, C. B. Schroeder, and S. M. Hooker, Nature Physic 2, 696 (2006).

[7] J. Ralph, K. Marsh, A. Pak, W. Lu, C. Clayton, F. Fang, W. Mori, and C. Joshi, Phys. Rev. Lett. 102 (2009).

[8] A. G. R. Thomas, Z. Najmudin, S. P. D. Mangles, C. D. Murphy, A. E. Dangor, C. Kamperidis, and K. L. Lancaster, Phys. Rev. Lett. 98, 095004 (2007).

[9] S. Kneip, S. R. Nagel, S. F. Martins, S. P. D. Mangles, C. Bellei, O. Chekhlov, R. J. Clarke, N. Delerue, E. J. Divall, G. Doucas, et al., Phys. Rev. Lett. 103, 035002 (pages 4) (2009).

[10] D. H. Froula and et. al., Phys. Rev. Lett. (in press) (2009).

[11] E. Esarey, P. Sprangle, J. Krall, and A. Ting, IEEE Trans. Plasma Science 2, 252 (1996).

[12] C. Decker, W. Mori, K.C., Tzeng, and T. Katsouleas, Phys. Plasmas 3, 2047 (1996).

[13] W. Lu, M. Tzoufras, C. Joshi, F. Tsung, W. Mori, Jiera, R. A. Fonseca, and L. Silva, Phys. Rev. ST Accel. Beams 10, 061301 (2007).

[14] J. Ralph, C. Clayton, K. Marsh, A. Pak, W. Lu, W. Mori, and C. Joshi, New Journal of Physics (in Preparation) (2010).

[15] W. Lu, C. Huang, M. Zhou, M. Tzoufras, F. Tsung, W. Mori, and T. Katsouleas, Phys. Plasmas 13, 056709 (2006).

[16] G.-Z. Sun, E. Ott, Y. Lee, and P. Guzdar, Phys. Fluids 30, 526 (1987).

[17] L. V. Keldysh, Sov. Phys. J. Exp. Theor. Phys. 20, 1307 (1965).

[18] B. Pollock, C. Clayton, L. Divol, S. Glenzer, C. Joshi, V. Leurent, K. Marsh, A. Pak, J. Palastro, J. Ralph, et al., in Proceedings from Particle Accelerator Conference 2009 (2009).

[19] S. Semushin and V. Malka, Rev. Sci. Instrum. 72, 2961 (2001).

[20] A. Pak, K. A. Marsh, S. F. Martins, W. Lu, W. B. Mori, and C. Joshi, Phys. Rev. Lett. (in press) (2009). 